\title{
Wide Area Surveillance using SSR Mode S Multilateration: advantages and limitations
}

\author{
Gaspare Galati ${ }^{1}$, Mauro Leonardi ${ }^{2}$, Pierfrancesco Magarò ${ }^{3}$, Valerio Paciucci ${ }^{4}$ \\ 1,2,3,4 Tor Vergata University, DISP and Vito Volterra Centre, Via del Politecnico 1, 00133, Rome, Italy,
}

Voice +390672597416 , Fax +390672597532

\begin{abstract}
There is an increasing interest on the various applications of the SSR Mode S ( Secondary surveillance radar-mode Selective) for air traffic and airport traffic management. This paper discusses the applications of ICAO standard Mode $S$ signals, in particular in the down link channel around $1090 \mathrm{MHz}$, to locate and identify cooperating targets in the airport using Multilateration (MLAT) and in the terminal area/en route using Wide Area Multilateration (WAM). Performance analyses and some comparison with the more traditional SSR system (ATC radar beacon system : ATCBRS) are carried out.
\end{abstract}

\section{INTRODUCTION}

The general interest in the applications using the ICAO standard SSR Mode S signals - specially in the downlink channel around $1090 \mathrm{MHz}$ - is more and more increasing for air traffic control and management (ATC/ATM), airport traffic management (A-SMGCS) and airborne collision avoidance (A-CAS/T-CAS).

Indeed, the are several surveillance, data link and identification systems using this standard, such as the traditional and well-known SSR (Secondary Surveillance Radar, Modes A/C and Mode S) [1], and the modern Multilateration and ADS-B [2], which are widely used all over the world.

In this context, the most recent applications for cooperative detection, location and identification of aircraft and equipped vehicles are based on the Multilateration system (MLAT) for airport surveillance and its ongoing version for terminal area/route surveillance (WAM, Wide Area MLAT).

MLAT systems consist of a number (e.g. 15, 20 or even more in complex layouts) of low-cost, receiving ground stations connected to a central processing subsystem where targets are located on the basis of (at least four) time of arrival measurements, by solving a system of non-linear equations.. The MLAT system functions and the recommended performance are described in [3].

The WAM main areas of applications are:

- 3D Surveillance systems for Control Zone /Terminal Area (CTR/TMA);

- En-route surveillance systems;

- Precision runway/approach monitoring (PRM);

- Integration of navigation, communications and surveillance by ADS (Automatic Dependent Surveillance) in areas with scarce ground infrastructures;

Its expected main operational benefits are:

- Independent height measurement (fully 3D systems);

- Non-rotating mechanical parts;

- Modularity - graceful degradation;

- ADS augmentation.
As several Multilateration Systems have been studied and installed for airport applications the aim of this work is to present a Wide Area Surveillance system as an extension of the surface MLAT. This could be a companion or even a potential alternative to widely used Secondary Surveillance Radar (SSR) stations as Air Traffic Control (ATC) surveillance systems.

A typical MLAT system as shown in Figure 1, is made up by a number (e.g. 12 to 20) of Measurement Stations capable of receiving, time-tagging and transmitting over a LAN the replies and the squitters (for short, R/S) present in the coverage area. Moreover, one or more Reference Transponders permit synchronization and monitoring of the whole system; the Times of Arrival (TOA's) of replies/squitters due to the SSR equipped aircraft and vehicles and to the reference Transponder(s) are processed in the Central Processing Station where multilateration algorithms locate the aircrafts and the mobiles. Different technical solutions have been implemented to locate the SSR transponder on the airport surface. The MLAT location error is affected by a significant Dilution of Precision (DOP), therefore in order to maintain the overall a low location rms error (in [3] it is specified an error below $7.5 \mathrm{~m}$ for $95 \%$ of time for surface surveillance) the rms error of each station has to be of the order of 1 meter, i.e. the various contributions (whose root sum squares is to be below $1 \mathrm{~m}$ ) must be kept in the order of 0.3 meters, corresponding to $1 \mathrm{~ns}$ of equivalent time error.

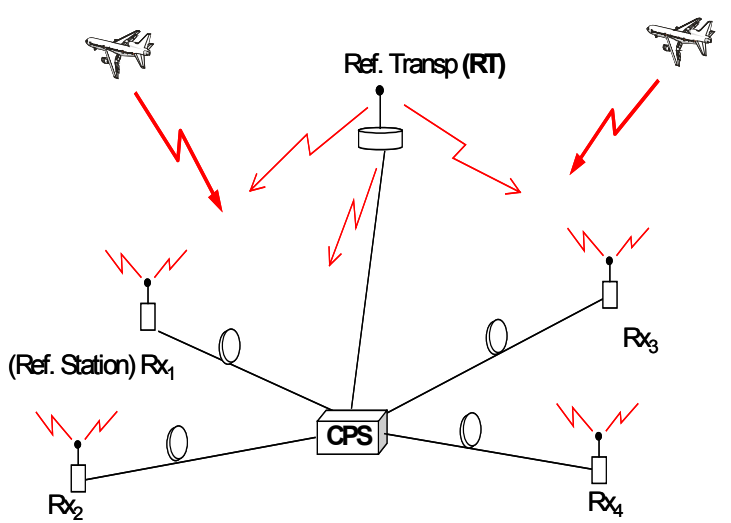

Fig. 1. MLAT Architecture. (CPS $=$ Central Processing Station, $\mathbf{R x}=$ Receiving station, $\mathbf{R T}=$ Reference Transponder $)$.

\section{MEASUREMENTS AND LOCATION TECHNIQUES IN MLAT}

In this section the classical leading/trailing-edge measurement, which is commonly used in the SSR context for TOA estimation, is compared to a novel 
[4],[5] more effective one, derived from the maximum likelihood estimation (MLE). The theoretical derivation is described in [5]. The novel approach adopts a digital filter matched to the SSR pulse waveform. The centroid of each pulse is estimated by the maximum of the matched filter output. This approach requires a wide band receiver (in this case a linear receiver, Bandwidth $=30 \mathrm{MHz}$ ) before the A/D conversion; pulse detection and optimal (in the MLE sense) measurement is performed by a FIR filter. The TOA performance are limited by errors due to a) interference, b) receiver noise, c) propagation phenomena (multipath, reflections), d) sampling, e) A/D converter dynamic range.

The TOA estimator is implemented by sending the received signal to a filter whose impulse response is equal to the derivative of the expected signal. The time of arrival is estimated by finding the instant in which the filter output crosses the zero value [5]. The interpolation of the two samples adjacent the null is performed at the output of the filter. The interpolation provides a measurement with an accuracy of a fraction of $T_{S}$ thereby reducing the sampling error $\sqrt{\left(T_{s}^{2} / 12\right)}$.

In the digital implementation, see Figure 2, assuming a sampling frequency of $60 \mathrm{Ms} / \mathrm{s}$, the estimator can be approximated by 18 sums and one difference.

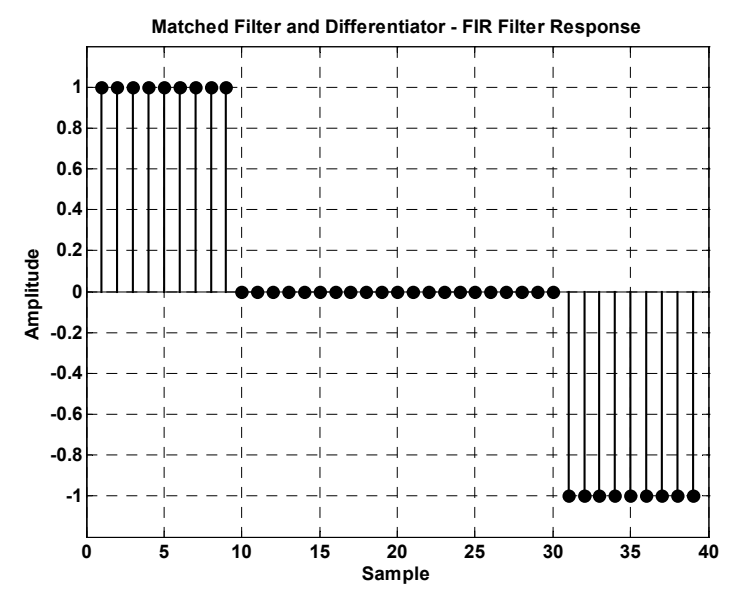

Fig. 2. TOA optimal estimator impulse response.

The r.m.s. error of TOA estimation is evaluated via simulation. In the trials the reception of standard ICAO pulses is simulated with a SNR in the range $20 \div 90 \mathrm{~dB}$ (corresponding to a distance of $50 \mathrm{Km}$ and $15 \mathrm{~m}$ respectively away from each sensor). In this work we propose an extension to a wider area w.r.t. the MLAT case (airport) studied in [5]. Figure 3 shows the rms error (vs. distance) of the MLE estimator compared to the results obtained with the traditional method. The following values have been assumed:

- $\quad$ receiver antenna gain $=6 \mathrm{dBi}$;

- $\quad$ receiver noise figure $=5.98 \mathrm{~dB}$;

- $\quad$ bandwidth=30 MHz;

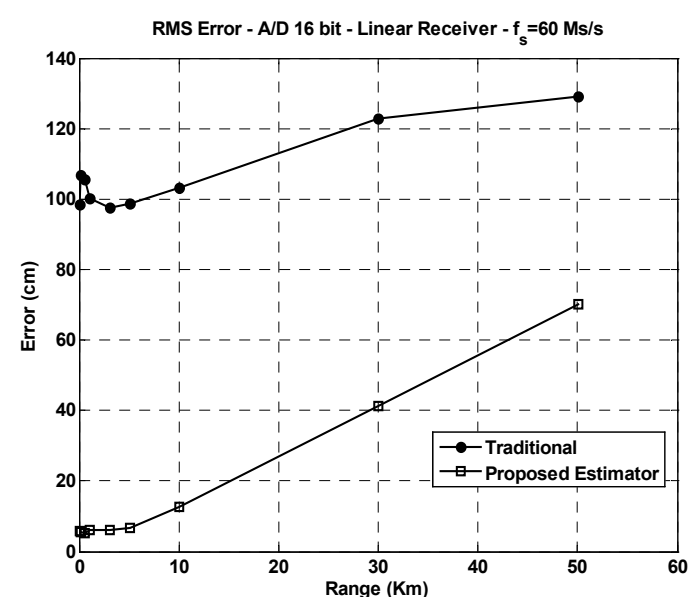

Fig. 3. Comparison of the RMS error $(\mathrm{cm})$ vs. distance $(\mathrm{Km})$ between the Traditional TOA estimator and the Proposed Method (Matched Filter plus differentiation followed by interpolation and zero-crossing).

In this extended configuration each receiving station has a coverage of about $50 \mathrm{Km}$; for longer range the performance decrease heavily and the estimation of the TOA is not assured.

The gain in the accuracy at $50 \mathrm{Km}$ is approximately $60 \mathrm{~cm}$. These values for the error are used in the localization simulations as the measurement error of each sensor.

\section{MULTILATERATION ALGORITHMS}

TOA measurements allow the TDOA computation and the definition of a set of equations which provide the target's position without the knowledge of the time of emission. The solution of these equations represents the intersection of M-1 hyperboloids, if the number of receiving stations is $\mathrm{M}$.

The range $d_{k}$ from the $k$-th sensor located at $\left(x_{k}, y_{k}, z_{k}\right)$ to the emitter $(x, y, z)$ is:

$$
d_{k}=\left[\left(x-x_{k}\right)^{2}+\left(y-y_{k}\right)^{2}+\left(z-z_{k}\right)^{2}\right]^{1 / 2}, k=1, \ldots M
$$

TDOA between receiver the $k$-th station and the reference station labeled as 1 can be written as:

$$
d_{k, 1}=c\left(t_{k}-t_{1}\right)=d_{k}-d_{1}, \quad k=2, \ldots M
$$

It has to be noted that the $d_{k, 1}$ elements are measured terms including additive measurement noise that can be assumed to be zero mean stationary Gaussian random process. The set of equations in (1) is a nonlinear vector function in source position coordinates.

There are different approaches to resolve this equations system.

\section{a) Closed form algorithm}

A possible close form method is presented in [6]. This approach approximates the ML estimator when the TDOA errors are reasonably small. The TDOA equations are transformed into another set of linear equations, assuming that there is no relation among $x, y$, $z$ and $d_{l}$ (distance between source and station 1 assumed as reference). Then the system can be solved by the Least Squares Method that gives a first solution, in case of far source, a second one if the source is close, and a final ambiguous solution introducing the known relation between the source position coordinates and $d_{l}$ (1). Only the final solution is ambiguous, like all the closed form procedures, whilst the initial and the intermediate ones are unique. 


\section{b) Iterative algorithm}

A possible solution is shown in [7], [8]. It is an iterative scheme to solve the algebraic non-linear equations. The method starts with a rough initial guess that is improved by minimizing the local linear least squared error. The most important drawbacks are the need of the initial guess, the convergence (not assured) and that the process is computationally burdensome. This method assumes that the linearization error is small. It as been illustrated in [9] that the higher order terms are significant in determining the solution in bad geometric dilution of precision (GDOP) situations. Even through the noise power is relative small there is no guarantee that the obtained solution is accurate. However this method presents some advantages [7]: a) Multiple independent measurements to a single station are averaged naturally; b) multiple measurements and mixed-mode (i.e non TOA, such as DOA...) measurements are combined properly, i.e. with the correct geometric factor, and can be weighted according to their a priori accuracies; c) The statistical spread of the solution can be found easily and naturally (DOP); d) failure to converge is easy to detect.

\section{c) Possible solutions}

In theory multilateration system equation with four or more sensor inputs can be used to compute three dimensional position; however, because, in many cases, sensors are ground based and do not provide sufficient elevation angle diversity, the vertical dilution of precision (VDOP) is usually too large to be useful for certain applications. In practice, only two-dimensional coordinates are calculated for aircraft surveillance with altitude obtained from decoding the $1090 \mathrm{MHz}$ aircraft transponder reply message. However an independent $3 \mathrm{D}$ positioning of transponder is possible and aircraft altitude can be set with an higher accuracy than using barometric altitude report if more suited measurements, e.g. of Direction of Arrival (DOA), are fused with TOA measurements to reconstruct the aircraft position.

Differently from surface applications (A-SMGCS), in Wide Area surveillance, some aspects, as clock synchronization and ambiguity resolution in source localization, are more critical.

Thus, in the present work, possible unambiguous methods for the resolution of the location equation system have been studied in order to obtain better performance in term of accuracy, convergence probability, number of iteration and computation burden.

A possible way for source location, using only TOA measurements, is to use the second unambiguous solution of the closed form algorithm, above-mentioned, as initial position for the iterative algorithm, as shown in figure 4.

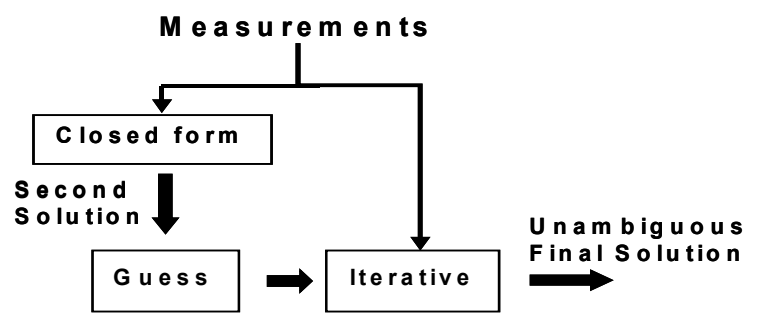

In this way it is possible to obtain a position estimation without ambiguity and with a minimum number of iterations.

In fact in Wide Area application the knowledge of a initial position for an iterative algorithm is even more necessary than the surface application in which the aircraft height is almost known and the limited area dimensions ensure a limited number of iterations.

Another solution using mixed TOA and DOA (Direction Of Arrival) measurements is being studied for aircraft position calculation. In case of bad VDOP the introduction of elevation angle measurements can substantially enhance the 2D and 3D accuracy even in case of low angle resolution antenna, as suggested in [10]. So it is possible, starting with the second solution of close form algorithm, to implement an iterative algorithm using TOA and DOA measurements [4],[7].

\section{CASE STUDY}

In this section the results of the position estimation by MLAT are presented. We have analyzed the performance of the proposed solution in an extended area around an airport surface. We started from the real configuration of the Marco Polo airport in Venice (buildings, walls, trellis, etc) where fourteen receiving stations at different heights $(0-40 \mathrm{~m})$ have been located, by a preliminary optimization of coverage and of precision, on the surface. More four remote stations have been added at about $20-25 \mathrm{Km}$ away from the center of the airport. The heights of these additional antennas are set in a range of $80-100 \mathrm{~m}$ to mitigate the high DOP values due to the airport-only configuration. Using an higher number of sensor will improve the geometry and dilution of precision of the solution and will increase the overall system capacity.

The considered area is $60 \mathrm{Km}$ length and $5 \mathrm{Km}$ wide with the airport in its center. It has to be noticed that the four stations outside the airport are most displaced in the left-right direction with respect to the airport, and closer to it in the up-down direction, as the considered landing path is left-right. With this layout (supposing all the eighteen antennas in visibility), a typical approaching trajectory has been reproduced with a $3^{\circ}$ inclination with respect to the $x-y$ plane of and the cells having dimensions $100 \mathrm{~m} \times 100 \mathrm{~m}$. At first HDOP and PDOP (Dilution Of Precision) have been calculated in deterministic way:

$$
P D O P=\sqrt{\text { trace }\left(\left(H^{T} \times Q^{-1} \times H\right)^{-1}\right)}
$$

$H$ is the Jacobian Matrix and $Q$ the classical matrix with 2 as diagonal elements and 1 elsewhere. The HDOP contains only the first two components $(x, y)$ without including $z$ component. In the following picture (figure 5) the airport is contained in the black belt (at approx. $30 \mathrm{Km}$ from the left). In the black vertical stripe (figure 5 and following) the DOP values and RMS errors have not been computed. The Horizontal DOP has a maximum level of about 20. Figure 5 also shows the position of the four stations outside the airport.

Fig. 4. Proposed location procedure. 


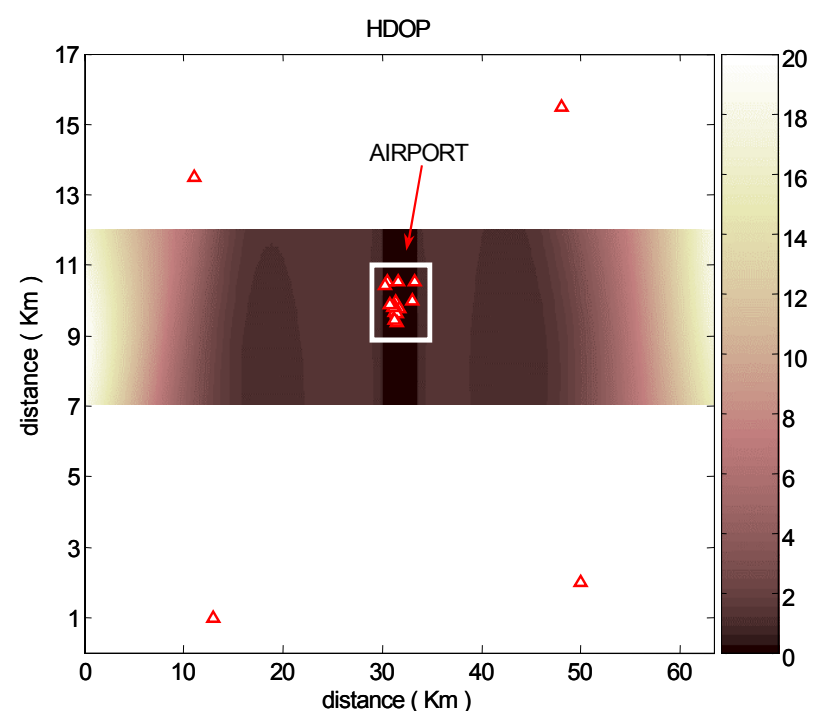

Fig. 5. Horizontal - DOP. The airport area lies at the center of the figure.

The PDOP parameter is shown in figure 6 (different $\mathrm{y}$-axis scale w.r.t. figure 5). These parameters give an idea of the geometrical goodness of the installation. The simulation of the position estimation provides the overall performance of the system, in term of position accuracy.

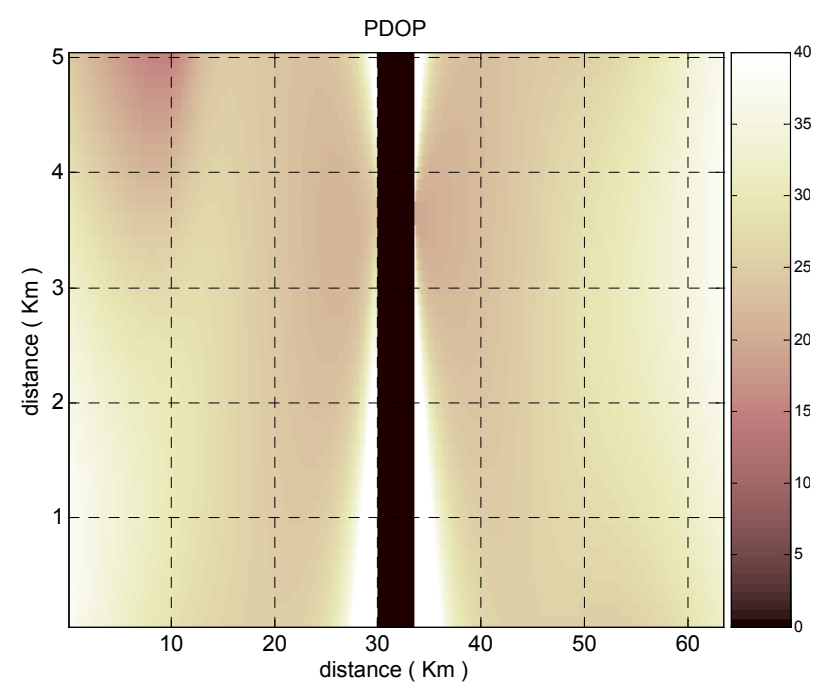

Fig. 6. Position - DOP.

A second step has been to use the mixed algorithm (figure 4) with the introduction of the additive TOA measurement noise (assumption: zero mean stationary Gaussian random process).

$$
\begin{aligned}
& r m s 3 D=P D O P \cdot \sigma_{T O A} \\
& r m s 2 D=H D O P \cdot \sigma_{T O A}
\end{aligned}
$$

However, for each trial, different noise powers have been used according to the distance target-station to simulate the TOA measurements, according to the error in figure 3. Thus the r.m.s. error cannot be expressed as (4) since there are different $\sigma_{T O A}$ depending on the receiving station.

So the performances of the mixed algorithm in terms of accuracy, number of iterations and convergence probability have been evaluated as follows.

The rms errors are shown in figure 7 . The algorithm computes the 3D ( $x, y, z$ coordinates are used) solutions for the location equations. The position error is that of figure 7 . The maximum level is 30 meters.

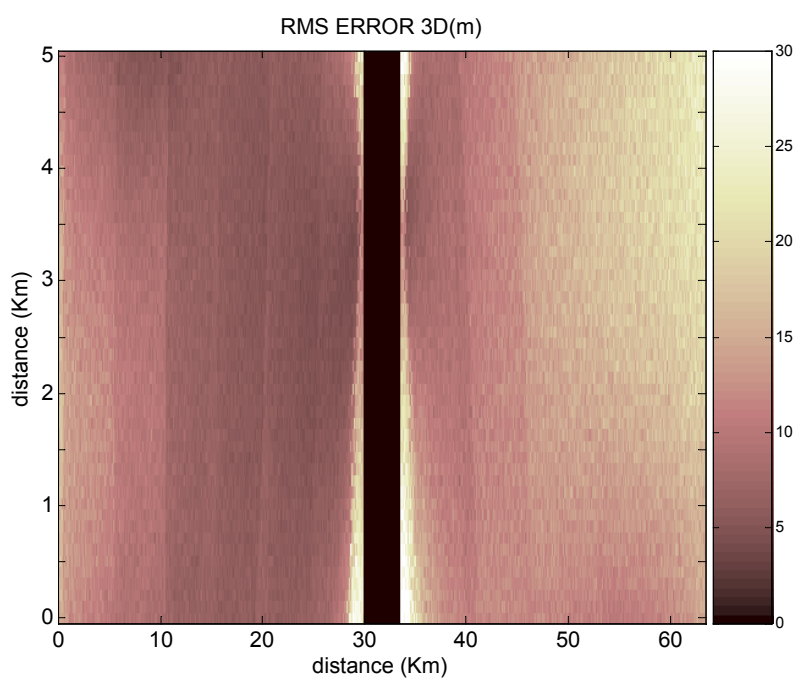

Fig. 7. RMS Error 3D (m), measurement error as in fig. 3.

Figure 8 shows the 2D error. This error is the difference between the projection of the estimated position $(x, y, z)$ in the $x, y$ plane and the real traget's position. This parameter - the 2D error - is used in comparison with the usual 2D SSR measurement (the height is acquired by the barometer). The maximum error is below 13 meters (at $30 \mathrm{Km}$ away from the airport).

SSR Mode S systems performs an azimuth accuracy of $0.066^{\circ} \mathrm{rms}$ (approx. $31 \mathrm{~m} @ 30 \mathrm{Km}$ ).

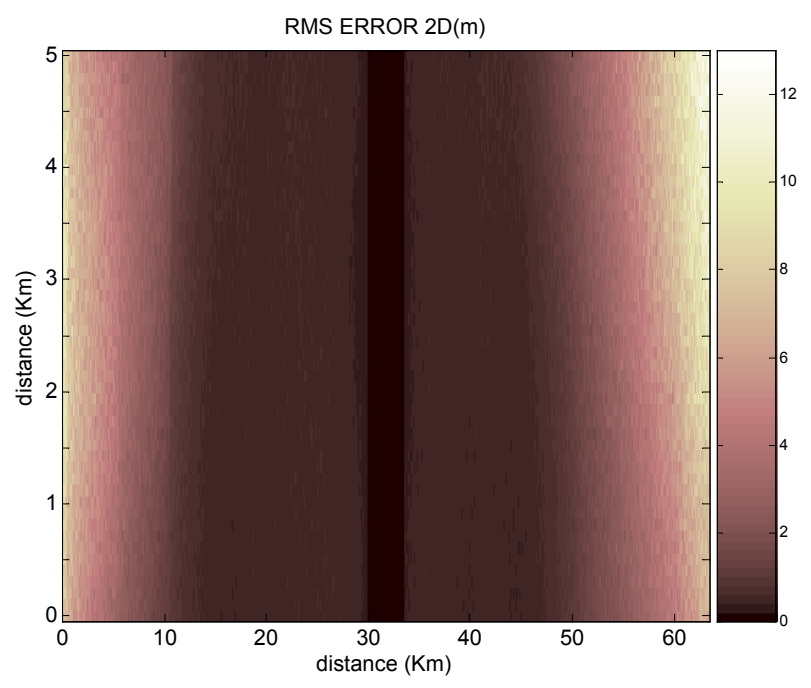

Fig. 8. RMS Error 2D (m), measurement error as in fig. 3.

These simulations also reveals that the average number of iterations (i.e. the number of steps required to reach the adequate threshold for position accuracy) is approximately 4 in the majority of the considered area.

The probability of convergence is above $98 \%$ in the considered area (except for negligible zones).

\section{WIDE AREA MULTILATERATION: ADVANTAGES AND LIMITATIONS}

Because of the distributed nature of multilateration sensors, it is only natural that interest would be growing to explore the use of the system in wide area applications, such as terminal area or en route surveillance, airport management, airport security, airline operations. For these types of applications an MLAT system has the potential of providing equivalent 
or higher levels of service at reduced cost when compared to traditional surveillance radar system. Moreover in hardly accessible areas and/or mountain the surveillance coverage by Secondary Surveillance Radar (SSR) is economically inconvenient (above all for low altitude coverage).

Multilateration is a potential solution. Earlier tests [2],[11] and analysis indicate that multilateration capabilities are comparable to those of SSR in some respects, and demonstrate the feasibility of multilateration systems for wide-area surveillance. When compared to the accuracy of terminal radar throughout a typical terminal area, these result represent an improvement in accuracy. Moreover, experience shows that track update rates from the multilateration system are significantly higher.

In wide-area applications, not only the number of sensors (likely to be more limited for cost reasons) concerns, but also the locations are constrained by logistic reasons, albeit to a lesser extent than erecting traditional surveillance radar buildings. An important issue is the resolution of the solution ambiguity, satisfying the TDOA equations, as it relates to track initiation performance and false track rate of the surveillance system.

A practical issue that distinguishes wide-area and airport surface applications is clock synchronization. Airport MLAT systems used a $1090 \mathrm{MHz}$ reference transponder to provide this function. However in wide area surveillance application, more reference transponder may be needed to cover all the receivers owing to the line-of-sight constrains. As all of these transponders have to be synchronized and have to be running at any time, system complexity increases significantly and hence cost.

\section{CONCLUSIONS}

The main advantages and problems of Wide Area Multilateration as applied to Air Traffic Control have been outlined. A preliminary assessment of accuracy has been done with comparisons with SSR. From the overall study it results that some technical and systemlevel choices as derived from airport MLAT can be applied to WAM, other require modifications.

The aim of a broader study is:

- assessment of the conditions in which the WAM solution is to be considered with respect to the classical Secondary Surveillance Radar;

- definition of the required upgrading for central processing station of WAM (multilateration algorithms, synchronization)

- definition of new system solutions (fusion of TOA with range and possibly with angular, i.e. DOA measurements) as well as of overall system improvements to fulfill the requirements for a next-generation Wide Area Surveillance System.

\section{REFERENCES}

[1] M. C. Stevens, "Secondary Surveillance Radar", Artech House, 1998.

[2] J.T.Beyer, "Stakeholder benefits of Wide Area Multilateration”, JISSA '05, Paris 20-21 June 2005.

[3] Eurocae ED-117 "Minimum operational performance specifications for Mode $\mathrm{S}$ multilateration systems for use in A-SMGCS", April 2003, available at Eurocae.

[4] G. Galati "Sistema di sorveglianza ad alta precisione mediante multilaterazione di segnali SSR", Italian patent RM 2004 A000249, Tor Vergata University, 17.05.2004 ; " High Precision Surveillance System by means of Multilateration of SSR Signals", Patent Cooperation Treaty Application PCT/IB 2005/51519 , subm. No. 4622, 10 May 2005, Applicant : Università degli Studi di Roma Tor Vergata.

[5] G. Galati, P. De Marco, L. Menè, M. Leonardi, P. Magarò, M. Gasbarra "New Time of Arrival Estimation Method for Multilateration Target Location”' JISSA '05, Paris 20-21 giugno 2005.

[6] Y.T. Chan, K.C.Ho, "A simple and efficient estimator for hyperbolic location", IEEE Transactions on Signal Processing, vol. 42, no. 8, Agosto 1999.

[7] Wade H. Foy, "Position-location solutions by Taylor series estimation," IEEE Transactions on Aerospace and Electronic Systems, vol. AES-12, no. 2, March 1976.

[8] Don J. Torrieri, "Statistical Theory of passive location systems,", IEEE Transactions on Aerospace and Electronic Systems, vol. AES-20, no. 2, March 1984.

[9] S. Bancroft, "An algebraic solution of the GPS equations", IEEE Transactions on Aerospace and Electronic Systems, vol. AES-21, pp. 56-59, Jan 1985.

[10] Pavel Bezousek, Vladimir Kubecek, "A 3D passive surveillance Vera System accuracy analysis, Microwaves, Radar and wireless communications, 2000, $13^{\text {th }}$ Mikon.

[11] Patrick J. Martone, George E. Tucker. "Candidate requirements for multilateration and ADS-B systems to serve as alternatives to secondary radar". Digital Avionics Systems, DASC, the $20^{\text {th }}$ Conference, vol 2, pp. 7c2/1- 7c2/12 October 2001. 
